\title{
Clinical Management of High and Very High Risk Patients with Hyperlipidaemia in Central and Eastern Europe: An Observational Study
}

\author{
Ivo Petrov · Andreea Dumitrescu • Michaela Snejdrlova • \\ Barak Zafrir · Beata Wożakowska-Kapłon · Lubomira Fabryova • \\ Hrvoje Pintarić · Ian Bridges · Reneta Petkova
}

Received: October 17, 2018 / Published online: February 13, 2019

(C) The Author(s) 2019

\begin{abstract}
Introduction: A retrospective/prospective observational study was conducted to explore the current management of hyperlipidaemia in high-risk (HR) and very high risk (VHR) patients in central/eastern Europe and Israel.
\end{abstract}

Enhanced digital features To view enhanced digital features for this article, including a slide deck summarising the study, go to https://doi.org/10.6084/ m9.figshare.7594700.

Electronic supplementary material The online version of this article (https://doi.org/10.1007/s12325019-0879-1) contains supplementary material, which is available to authorized users.

\section{Petrov $(\varangle)$}

University Clinic of Cardiology, Angiology and

Electrophysiology, City Clinic, Cardiovascular

University Centre, University of Sofia, Sofia,

Bulgaria

e-mail: petrovivo@hotmail.com

\section{A. Dumitrescu}

CardioPrevent Foundation, University of Medicine and Pharmacy, Timisoara, Romania

\section{Snejdrlova}

Centre of Preventive Cardiology, IIIrd Department of Internal Medicine, Charles University in Prague, General University Hospital, Prague, Czech Republic

\section{B. Zafrir}

Department of Cardiovascular Medicine, Lady Davis Carmel Medical Centre, Haifa, Israel
Methods: The study enrolled adult patients who were receiving lipid-lowering therapy and attending a specialist (cardiologist/diabetologist/lipidologist) or internist for a routine visit at 57 sites (including academic/specialist/internal medicine centres) across Bulgaria, Croatia, Czech Republic, Israel, Poland, Romania and Slovakia. Data were collected from medical records, for the 12 months before enrolment, with/without $\leq 6$ months' additional prospective follow-up.

Results: A total of 1244 patients, mean (SD) age 63.3 (11.3) years were included (307 with familial hypercholesterolaemia (FH), 943 secondary prevention patients). Almost all patients $(98.1 \%)$ were receiving statins $(76.7 \%$ monotherapy $/ 21.4 \%$ combined therapy), with

B. Wożakowska-Kapłon

Cardiology Clinic, Jan Kochanowski University in

Kielce, Kielce, Swietokrzyskie, Poland

L. Fabryova

MetabolKLINIKsro, Department for Diabetes and Metabolic Disorders, Lipid Clinic, MED PED Centre, Bratislava, Slovakia

H. Pintarić

Department of Internal Medicine, Faculty of

Dentistry, University of Zagreb, Zagreb, Croatia

I. Bridges

Amgen Ltd, Cambridge, UK

R. Petkova

Amgen Bulgaria EOOD, Sofia, Bulgaria 
53.1\% receiving high-intensity statin therapy: 127 patients (10.2\%) had adverse events attributed to statin intolerance. Mean (SD) low density lipoprotein cholesterol (LDL-C) levels were $3.3(1.7) \mathrm{mmol} / \mathrm{L}$ at the first, and 2.7 (1.3) $\mathrm{mmol} / \mathrm{L}$ at the last, visit of the retrospective phase of observation, with little change during the prospective phase. Less than one-quarter (23.8\%; 95\% CI $17.29-31.45 \%$ ) of HR patients and less than half $(42.0 \% ; 39.05-44.98 \%)$ of VHR patients achieved their risk-based LDL-C targets of $<2.5$ and $<1.8 \mathrm{mmol} / \mathrm{L}$, respectively. Less than $15 \%$ of $\mathrm{FH}$ patients reached these targets $(10.9 \%(5.6-18.7 \%)$ of HR and $12.1 \%$ (8.0-17.4\%) of VHR patients). The revised 2016 ESC/EAS target for HR patients $(2.6 \mathrm{mmol} / \mathrm{L})$ was met by $28.5 \%$ (21.44-36.38\%) of HR patients overall. Almost one-half of patients (42.1\%) experienced one or more cardiovascular events during observation.

Conclusion: Our findings confirm that, despite widespread statin use, a substantial proportion of patients treated for hyperlipidaemia in central/ eastern Europe and Israel, particularly those with $\mathrm{FH}$, do not reach recommended LDL-C targets, thus remaining at risk of cardiovascular events.

Funding: Amgen (Europe) GmbH.

Keywords: Cardiology; Cardiovascular events; Hyperlipidaemia; Low-density lipoprotein cholesterol

\section{INTRODUCTION}

Hyperlipidaemia is a major modifiable risk factor for the development of cardiovascular (CV) disease, the leading cause of death and disability in the developed world [1]. It is estimated that up to $50 \%$ of the European population in the 35-64 years age bracket has total cholesterol level $>6.5 \mathrm{mmol} / \mathrm{L}$ [2]. A large body of data from both primary and secondary prevention settings demonstrate that reducing elevated total cholesterol, non-high-density lipoprotein cholesterol (non HDL-C), and most importantly low density lipoprotein cholesterol (LDL-C), via lipid-modifying treatment (LMT), lowers the risk of CV events [3-5]. Moreover, the degree of risk reduction is proportional to the LDL-C reduction. A meta-analysis of 21 randomised controlled trials of statin therapy in almost 170,000 patients indicated that for every $\sim 1 \mathrm{mmol} / \mathrm{L}$ reduction in LDL-C the risk of major vascular events is reduced by $\sim 20 \%$ over a period of approximately 5 years [6].

Guidelines from the European Society of Cardiology/European Atherosclerosis Society (ESC/EAS) emphasise the importance of setting clear LDL-C targets for patients with hyperlipidaemia and using aggressive LMT to achieve these [7, 8]. In 2011 the guidelines recommended an LDL-C goal of $<2.5 \mathrm{mmol} / \mathrm{L}$ $(96.5 \mathrm{mg} / \mathrm{dL})$ for high-risk (HR) patients and $<1.8 \mathrm{mmol} / \mathrm{L}(70 \mathrm{mg} / \mathrm{dL})$ for very high risk (VHR) patients. The target for HR patients was subsequently revised to $<2.6 \mathrm{mmol} / \mathrm{L}(100 \mathrm{mg} /$ dL) in 2016 [8]. Statin therapy has been the mainstay of hyperlipidaemia treatment for several decades. Guidelines recommend administration of the highest tolerated statin dose until the LDL-C target is met, with the addition of non-statin treatment(s) for patients failing to reach target with statin monotherapy $[7,8]$.

Most of the literature on LMT use and lipid control comes from 'Western' countries such as the USA and western Europe, with corresponding data from central and eastern European populations being much more limited, reflecting a lack of patient registries and access to public/health insurance data in eastern Europe. Rates of CV morbidity and mortality are generally much higher in eastern Europe than in western Europe [9], with 2015 data indicating that Lithuania had the highest standardized death rate for ischaemic heart disease in the European Union (760 per 100,000 male residents), followed by Latvia, Hungary, Slovakia, the Czech Republic and Romania (395-600 per 100,000 male residents, cf. $77 / 100,000$ in France) [10]. Thus, it is important to obtain more data from patients receiving hyperlipidaemia treatment in this region.

Accordingly, we conducted an observational study with the aim of gaining insight into the current management of hyperlipidaemia and LDL-C target achievement in HR and VHR patients in Bulgaria, Croatia, Czech Republic, Poland, Romania, Slovenia and Slovakia, as well as Israel. 


\section{METHODS}

\section{Study Population}

This retrospective/prospective observational study enrolled adult patients with hyperlipidaemia (HR or VHR as defined by 2011 ESC/EAS guidelines) [7] who were receiving LMT and attending a specialist (cardiologist/diabetologist/lipidologist) and/or internist for a routine visit during the enrolment period. They were required to have $\geq 2$ recorded LDL-C values and valid LMT documentation (type of medication and dose) for the retrospective data collection period. Patients receiving treatment for hyperlipidaemia within the course of an interventional clinical trial were excluded. All patients participating in the prospective phase were required to have all LDL-C and corresponding LMT data for this period.

\section{Study Conduct}

In order to enrol a representative patient population in the study, we included academic and non-academic centres, specialised clinics and internal medicine clinics. The study enrolled all eligible subjects who provided informed consent (as applicable per respective country legislation) from the participating sites.

Data were collected from individual patient medical records, for the 12 months prior to enrolment (retrospective phase) and $\leq 6$ months following enrolment in the three countries participating in the prospective phase (Romania, Poland, Czech Republic).

\section{Ethics/Consent}

There were no study-related medical procedures undertaken. The study was conducted in accordance with the Helsinki Declaration of 1964, as revised in 2013, and with countryspecific legal and regulatory requirements. It was approved by ethics committees and for registration or classification by regulatory bodies, as applicable in each country:

1. Bulgaria: Republic of Bulgaria Ministry of Health Ethics Committee for Multicentre Trial,
Sofia; 2. Croatia: Agency for Medicinal Products and Medical Products Central Ethics Trust, Zagreb; 3. Czech Republic: Ethics Committee of: Faculty Hospital Hradec Králové, Králové; Institute for Clinical and Experimental Medicine (IKEM) and Thomayer Hospital, Prague; Faculty Hospital Brno, Brno; Faculty Hospital Plzeň, Plzeň; General Teaching Hospital in Prague, Prague; St. Anne's Faculty Hospital in Brno, Brno; Ethics Committee for Multicentre Clinical Assessment of the Motol University Hospital, Prague; Pardubice Regional Hospital Svitavska Hospital 4. Israel: Helsinki Committees of: Edith Wolfson Medical Centre, Tel Aviv; Chaim Sheba Medical Center, Tel Hashomer; Hadassah Medical Center, Jerusalem; Rabin Medical Center, Petah Tikva; Lady Davis Carmel Medical Center, Haifa; Soroka Medical Center, Beersheba; Bnai Zion Medical Center, Haifa; Meir Medical Center, Kfar Saba; Ziv Medical Center, Safed. 5. Poland: Bioethical Commission in Kielce at the Świętokrzyska Medical Chamber. 6. Romania: Romania Medical Sciences Academy, National Bioethics Committee for Medicines and Medical Devices, Bucharest. 7. Slovakia: Multicentre Ethics Committee of Kosice SelfGoverning Region, Kosice.

The study followed generally accepted research practices described in Good Epidemiological Practice guidelines issued by the International Epidemiological Association. All data were handled in strictest confidence in conformity with national and European data protection regulations (such as Directive 95/46/EC). All patients provided written informed consent, where required by local regulations.

\section{Objectives}

The primary study objective was to estimate the proportion of patients achieving LDL-C levels of $<2.5 \mathrm{mmol} / \mathrm{L}$ and $<1.8 \mathrm{mmol} / \mathrm{L}$, the ESC/EASdefined target levels at the time for HR and VHR patients, respectively [7]. We also included the revised 2016 target of $<2.6 \mathrm{mmol} / \mathrm{L}$ for $\mathrm{HR}$ patients [8] in our analysis. Secondary objectives included LDL-C levels over time; use of statins/other LMT (type, dose, frequency) over time; patient clinical characteristics 
(demographics, medical history including familial hypercholesterolaemia (FH) diagnosis, statin intolerance symptoms (as recorded by the investigators on the case report form), CV events and hospitalisations).

The definition of statin intolerance is a matter of debate. For instance it has been defined by an International Lipid Expert Panel as 'the inability to tolerate at least two statins: one statin at the lowest starting daily dose and another statin at any daily dose, due to either objectionable symptoms (real or perceived) or abnormal laboratory determinations, which are temporally related to statin treatment and reversible upon statin discontinuation' [11]. However in this report the term statin intolerance simply refers to adverse events that were attributed to statin intolerance (partial or complete). CV events included coronary heart disease (unstable angina pectoris/myocardial infarction/sudden cardiac death), cerebrovascular disease (transient ischaemic attack/stroke) or peripheral artery disease (intermittent claudication/ischaemic rest pain/gangrene/abdominal aortic aneurysm/atrial fibrillation/heart failure/coronary revascularisation). Patients' CV risk was categorised according to physician evaluation. No formal hypothesis was tested.

\section{Study Sample Size}

In order to enable sufficiently precise estimates of the primary outcome measure (achievement of ESC/EAS LDL-C targets) to be calculated for each country, it was planned to enrol approximately 1300 subjects in total across eight countries, with each country enrolling between 80 and 150 subjects. Assuming that 50\% of subjects achieve the target, a sample size of 80 patients would enable the percentage to be estimated with a 95\% confidence interval (CI) with a half-width of $11.0 \%$, while a sample size of 150 patients would result in a half-width of $8.0 \%$.

\section{Data Analysis}

Summary statistics only are presented. For categorical variables, including the primary outcome measure, the frequency and percentage in category, with $95 \% \mathrm{CI}$, were calculated. For continuous variables the number of subjects, mean, median, standard deviation or standard error, 25th and 75th percentile (Q1, Q3), minimum and maximum are presented. There was no imputation for missing data.

LDL-C levels were analysed by patient subgroups of interest: $\mathrm{FH}$, secondary prevention, diabetic, ST-segment elevation myocardial infarction (STEMI) and statin intolerant (SI; see "Methods" section), as noted on the case report form. A post hoc analysis was conducted, comparing last-visit LDL-C levels for each subgroup with values for all other patients, using the nonparametric Mann-Whitney $U$ test.

For countries with retrospective and prospective data collection, the retrospective and prospective data were combined in the primary endpoint analysis. The two portions of data were also summarised separately, to evaluate any differences in terms of data quality and completion.

\section{RESULTS}

\section{Study Population}

A total of 1281 patients were enrolled at 57 sites, in Romania ( $n=239$ : 10 centres); Bulgaria (234: 8); Czech Republic (209: 11); Israel (205: 9); Poland (152: 6), Slovakia (152: 9) and Croatia (90: 4). The Slovenian centres did not participate because of delayed regulatory approval and this led to higher patient accrual rates in the other countries. Most sites were cardiology or diabetology/lipidology clinics: only the Czech and Slovak Republics included internal medicine clinics.

Thirty-seven patients were found to be ineligible and excluded from study participation, leaving 1244 patients who were followed retrospectively, with 585 also followed prospectively, between January 2015 and March 2017. Only seven patients discontinued study participation, due to death (4 patients), loss to follow-up (2) or subject decision (1) (Table S1; in electronic supplementary files). 
Table 1 Characteristics of the study population $(n=1244)$

\begin{tabular}{|c|c|c|}
\hline \multirow{2}{*}{$\frac{\text { Parameter }}{\text { Male/female }}$} & \multicolumn{2}{|l|}{ Category/statistic } \\
\hline & $n / n$ & $787 / 457$ \\
\hline \multirow[t]{3}{*}{ Age (years) } & Mean (SD) & $63.3(11.28)$ \\
\hline & Median (Q1-Q3) & $64.0(58.0-71.0)$ \\
\hline & Range & $18-92$ \\
\hline \multirow{3}{*}{$\begin{array}{l}\text { Body weight } \\
(\mathrm{kg})\end{array}$} & Mean (SD) & $84.5(16.59)$ \\
\hline & Median (Q1-Q3) & $83.0(73.5-94.0)$ \\
\hline & Range & $42-173$ \\
\hline \multirow{5}{*}{$\begin{array}{l}\text { Time since } \\
\text { diagnosis }^{\mathrm{a}}\end{array}$} & $<1$ year & $220(17.7 \%)$ \\
\hline & $\geq 1$ and $<2$ years & $81(6.5 \%)$ \\
\hline & $\geq 2$ and $<5$ years & $131(10.5 \%)$ \\
\hline & $\geq 5$ years & $534(42.9 \%)$ \\
\hline & Unknown & $278(22.3 \%)$ \\
\hline \multirow[t]{5}{*}{ Subgroup } & $\mathrm{FH}$ & $307(24.7 \%)$ \\
\hline & $\begin{array}{l}\text { Secondary } \\
\text { prevention }\end{array}$ & $943(75.8 \%)$ \\
\hline & Diabetes & $528(42.4 \%)$ \\
\hline & STEMI & $208(16.7 \%)$ \\
\hline & Statin intolerant ${ }^{\mathrm{b}}$ & $127(10.2 \%)$ \\
\hline \multirow{4}{*}{$\begin{array}{l}\text { DLCN score } \\
\text { (FH patients) }\end{array}$} & $<3$ & $51(16.3 \%)$ \\
\hline & $3-5$ & $86(27.5 \%)$ \\
\hline & $6-7$ & $86(27.5 \%)$ \\
\hline & $\geq 8$ & $84(26.8 \%)$ \\
\hline \multirow[t]{2}{*}{ CV risk } & High & $151(12.1 \%)$ \\
\hline & Very high & 1093 (87.9\%) \\
\hline
\end{tabular}

DLCN Dutch Lipid Clinic Network criteria [12]: definite/probable $\mathrm{FH} \geq 6$; possible $\mathrm{FH} 3-5$; unlikely $\mathrm{FH}$ $<3$; FH Familial hypercholesterolaemia; STEMI ST-Elevation Myocardial Infarction

${ }^{a}$ Diagnosis of hyperlipidaemia

b Patients with adverse events attributed to statin intolerance
Table 2 Summary of mono and combined lipid-modifying therapies (LMT), overall and for the familial hypercholesterolaemia $(\mathrm{FH})$ subgroup

\begin{tabular}{lcc}
\hline Treatment $^{\mathbf{a}}$ & \multicolumn{2}{l}{ No. of patients (\%) } \\
\cline { 2 - 3 } & Overall & FH \\
\hline Monotherapy & $954(76.7 \%)$ & $234(76.2 \%)$ \\
Statin & $10(0.8 \%)$ & $1(0.3 \%)$ \\
Other LMT & $7(0.6 \%)$ & $2(0.7 \%)$ \\
Ezetimibe & & \\
Combination therapy & $132(10.6 \%)$ & $50(16.3 \%)$ \\
Statin + ezetimibe & $107(8.6 \%)$ & $11(3.6 \%)$ \\
Statin + other LMT & $27(2.2 \%)$ & $9(2.9 \%)$ \\
Statins + other & & \\
LMT + ezetimibe & $7(0.6 \%)$ & $0(0 \%)$ \\
Other LMT + ezetimibe & $1244(100 \%)$ & $307(100 \%)$ \\
Total &
\end{tabular}

${ }^{a}$ Anytime during the observation period. Each subject is included only once (e.g. if they received statin monotherapy for a portion of the observation period and statin + ezetimibe for another portion, they are included under statin + ezetimibe)

Patient characteristics are summarised in Table 1 and lifestyle risk factors in Table S2. Mean patient age was 63.3 (SD 11.3) years and the majority (63\%) were men; duration of hyperlipidaemia ranged from $<1$ year $(17.7 \%)$ to $\geq 5$ years $(42.9 \%)$. Most $(943 ; 75.8 \%)$ were secondary prevention patients, with almost 90\% classified as VHR (1093; 87.9\%). There were 307 patients $(24.7 \%)$ with $\mathrm{FH} ; 528(42.4 \%)$ with diabetes and 208 (16.7\%) with STEMI, with 127 $(10.2 \%)$ being classified as statin intolerant (Table 1), as per "Methods" section.

Among those listed as having FH $(n=307)$, just over half had 'probable' or 'definite' $\mathrm{FH}$ (score $\geq 6 ; n=170 ; 55.4 \%$ ), according to Dutch Lipid Clinic Network (DLCN) criteria [12]. It is unclear whether FH was diagnosed clinically or genetically and whether the DLCN score was based on highest untreated LDL-C levels. 
Table 3 Summary of statin use at the first visit of the observation period

\begin{tabular}{llc}
\hline Statin & Dose (mg) & Patients (\%) \\
\hline Atorvastatin & $5-10$ & $98(7.9 \%)$ \\
& $20-30$ & $219(17.7 \%)$ \\
& $40-80$ & $311(25 \%)$ \\
Rosuvastatin & 5 & $23(1.9 \%)$ \\
& $10-15$ & $195(15.7 \%)$ \\
Simvastatin & $20-40$ & $194(15.6 \%)$ \\
Fluvastatin & $5-40$ & $86(6.9 \%)$ \\
Pravastatin & $40-80$ & $8(0.6 \%)$ \\
Lovastatin & $10-40$ & $8(0.6 \%)$ \\
Unknown/not taking statins & - & $3(0.2 \%)$ \\
Total & $10-40$ & $99(8.0 \%)$ \\
\hline
\end{tabular}

\section{Lipid-Modifying Therapies}

As shown in Table 2, almost all patients received statins during observation: 954 (76.7\%) as monotherapy and $266(21.4 \%)$ as part of combination therapy. A total of 159 patients $(12.8 \%)$ were taking a statin-ezetimibe combination, with/without other LMT. Statin dosage details were available for 1211 patients: 66/142 HR (46.5\%) and 577/1069 VHR patients (54.0\%) were taking high-intensity statin therapy (atorvastatin $40-80 \mathrm{mg}$ or rosuvastatin 20-$40 \mathrm{mg} /$ day) at some point during observation. Table 3 summarises statin type and dosages at first visit. Non-statin LMT is listed in Table S3: as well as ezetimibe $(n=167 ; 13.4 \%)$, this included fibric acid derivatives (117; 9.4\%), proprotein convertase subtilisin/kexin type 9 inhibitors (PCSK9i; 26; 2.1\%), bile acid-binding resins $(1 ; 0.1 \%)$ and niacin $(1 ; 0.1 \%)$.

\section{LDL-C Levels and Targets}

Mean (SD) LDL-C levels tended to be lower at the latest visit than at the earliest visit of the retrospective period in all patient subgroups: $3.3(1.7) \quad(n=1244)$ versus $2.7 \quad(1.3) \mathrm{mmol} / \mathrm{L}$ $(n=1205)$ overall (Table S4; Fig. 1$)$. Not unexpectedly, the $\mathrm{FH}$ subgroup had the highest mean (SD) LDL-C levels, at 5.1 (1.7) and 3.8 (1.5) $\mathrm{mmol} / \mathrm{L}$, respectively (Fig. 1). Patients with DLCN score 3-5 had slightly higher mean LDL-C than those with score $\geq 6$. During the prospective period there was little change in mean LDLC (Table S4). Comparison of mean LDL-C levels at the last visit showed the FH subgroup to have significantly higher, and the secondary prevention and diabetic subgroups to each have

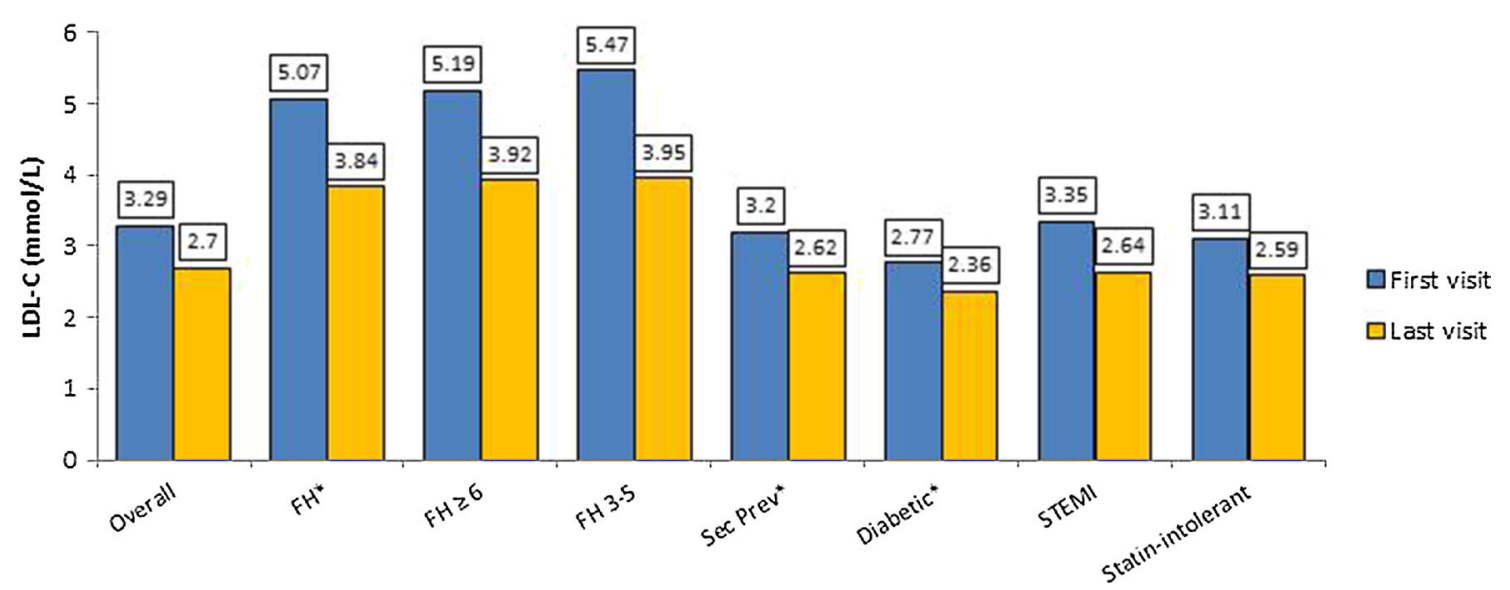

Fig. 1 Mean LDL-C levels, overall and by subgroup at the first and last visit of the retrospective period $(n=1244$ first visit; 1204 last visit). FH, Familial hypercholesterolaemia; $\mathrm{FH} \geq 6$ and FH 3-5 refer to DLCN scores [12];
SI, patients with symptoms attributed to statin intolerance. For subgroup numbers, see Table S3. ${ }^{*} P<0.001$ for last visit values versus rest of study population (Mann-Whitney $U$ test) 


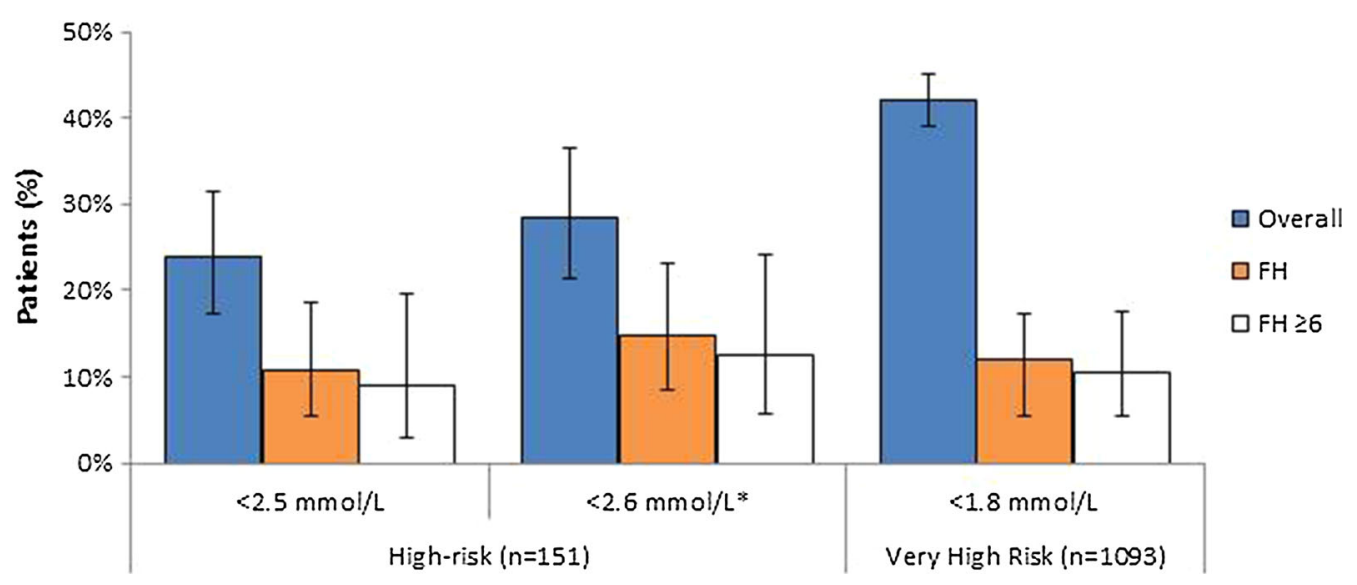

Fig. 2 Proportion of high-risk $(n=151)$ and very high risk $(n=1093)$ patients achieving ESC/EAS-defined LDL-C target at any time during the observation period;

significantly lower, levels than the remainder of the study population $(p<0.001$ all comparisons).

Achievement of risk-based ESC/EAS LDL-C targets (overall and $\mathrm{FH}$ patients) is shown in Fig. 2 . In the HR group, $36 / 151$ patients $(23.8 \%$; 95\% CI 17.29-31.45\%) achieved $<2.5 \mathrm{mmol} / \mathrm{L}$ and $43(28.5 \% ; 21.44-36.38 \%)$ achieved the current target of $<2.6 \mathrm{mmol} / \mathrm{L}$, at some point during the observation period. Among the VHR patients, $459 / 1093 \quad(42.0 \% ; \quad 39.05-44.98 \%)$ reached their target of $<1.8 \mathrm{mmol} / \mathrm{L}$ (Fig. 2). Post hoc analysis revealed that among the 577 VHR patients taking high-intensity statin treatment during the study, $261 \quad(45.2 \%$; 41.1-49.4\%) patients reached their LDL-C goal versus 189 (38.4\%; 34.1-42.9\%) of those who did not receive high-intensity statins (Table S5). Approximately $60-70 \%$ of HR and $60 \%$ of VHR patients who did not reach their target were not taking high-intensity statins.

There was a higher rate of target achievement in the prospective versus the retrospective phase, consistent with the LDL-C levels seen in these periods (Table S6).

As summarised in Table S5, very few FH patients reached their LDL-C target: 10.9\% (5.6-18.7\%) of HR and $12.1 \%(8.0-17.4 \%)$ of VHR patients attained $<2.5$ and $<1.8 \mathrm{mmol} / \mathrm{L}$, respectively, with $14.9 \%$ of $\mathrm{HR}$ patients (8.6-23.3\%) attaining $<2.6 \mathrm{mmol} / \mathrm{L}$. In those overall and for patients with $\mathrm{FH}$ (all FH patients and those with DLCN score $\geq 6$ ). ${ }^{*}$ Updated target in 2016 guidelines [8]

with definite/probable FH (DLCN score $\geq 6$ ), 5/56 (8.9\%; 3.0-19.6\%) HR patients reached $<2.5 \mathrm{mmol} / \mathrm{L}$ and $7 / 56 \quad(12.5 \%$; $5.2-24.17 \%)$ achieved $<2.6 \mathrm{mmol} / \mathrm{L}$, while 12/114 (10.5\%; 5.6-17.7\%) VHR patients achieved $<1.8 \mathrm{mmol} / \mathrm{L}$. Similar results were seen in those with DLCN score 3-5.

\section{Cardiovascular Events}

As summarised in Table 4, 910 patients (73.2\%) had a history of CV events ( $n=1-6$ events) at the start of observation and 523 patients (42.1\%) experienced events $(n=1-8)$ during observation. In total 37/151 (24.5\%) HR and 906/1093 (82.9\%) VHR patients (total 943; $75.8 \%$ ) experienced CV events prior to, or during, the observation period. (For a list of CV events that were captured in the study, see "Methods".)

\section{Adverse Events and Hospitalisations}

At enrolment, 127 patients (10.2\%) were classified as statin intolerant; the most common symptom being statin-associated muscle symptoms (SAMS) as defined by an EAS Consensus Panel [13] (7.8\% of patients overall). Other symptoms included hepatotoxicity, new-onset diabetes mellitus and other rare statin-associated adverse events $(<1 \%$ of patients overall). 
Table 4 Summary of cardiovascular events

\begin{tabular}{|c|c|c|}
\hline \multirow[t]{2}{*}{ Number of events ${ }^{a}$} & \multicolumn{2}{|l|}{ Patients (\%) } \\
\hline & $\begin{array}{l}\text { Overall } \\
(n=1244)\end{array}$ & $\begin{array}{l}\text { FH } \\
(n=307)\end{array}$ \\
\hline \multicolumn{3}{|c|}{ Before start of observation (historical events) } \\
\hline 1 & $487(39.1 \%)$ & $109(35.5 \%)$ \\
\hline 2 & $275(22.1 \%)$ & $41(13.4 \%)$ \\
\hline 3 & $99(8.0 \%)$ & $18(5.9 \%)$ \\
\hline 4 & $37(3.0 \%)$ & $6(2.0 \%)$ \\
\hline 5 & $9(0.7 \%)$ & $1(0.3 \%)$ \\
\hline 6 & $3(0.2 \%)$ & $0(0 \%)$ \\
\hline Total with events & $910(73.2 \%)$ & $175(57.0 \%)$ \\
\hline \multicolumn{3}{|l|}{ During observation } \\
\hline 1 & $251(20.2 \%)$ & $75(24.4 \%)$ \\
\hline 2 & $196(15.8 \%)$ & $77(25.1 \%)$ \\
\hline 3 & $56(4.5 \%)$ & $18(5.9 \%)$ \\
\hline 4 & $13(1.0 \%)$ & $4(1.3 \%)$ \\
\hline 5 & $2(0.2 \%)$ & $0(0.0 \%)$ \\
\hline 6 & $0(0.0 \%)$ & $0(0.0 \%)$ \\
\hline 7 & $2(0.2 \%)$ & $0(0.0 \%)$ \\
\hline 8 & $3(0.2 \%)$ & $0(0.0 \%)$ \\
\hline Total with events & $523(42.1 \%)$ & $174(56.7 \%)$ \\
\hline
\end{tabular}

${ }^{a}$ Coronary heart disease (unstable angina pectoris/myocardial infarction/sudden cardiac death), cerebrovascular disease (transient ischaemic attack/stroke) or peripheral artery disease (intermittent claudication/ischaemic rest pain/gangrene/abdominal aortic aneurysm/atrial fibrillation/heart failure/coronary revascularisation)

A total of 528 patients (42.4\%) were hospitalised at least once during the observation period. Reasons included coronary revascularisation ( $n=119 ; 9.6 \%$ of patients); heart failure (71; 5.7\%); unstable angina pectoris $(69 ; 5.6 \%)$, or STEMI, non-STEMI, atrial fibrillation, intermittent claudication, ischaemic pain at rest $(1-5 \%)$.
Table 5 Most common reasons for switching/modifying/ discontinuing lipid-modifying therapy (includes all reasons applying to $>5$ patients in the overall group)

\begin{tabular}{|c|c|c|}
\hline \multirow[t]{2}{*}{ Status and reason ${ }^{a}$} & \multicolumn{2}{|l|}{$n(\%)$} \\
\hline & Overall & FH \\
\hline $\begin{array}{l}\text { Switched to other } \\
\text { LMT (incl. } \\
\text { statins) }\end{array}$ & $163 / 1244(13.6 \%)$ & $25 / 307(8.1 \%)$ \\
\hline $\begin{array}{l}\text { Insufficient lipid- } \\
\text { lowering effect }\end{array}$ & $83 / 163(50.9 \%)$ & $17 / 25(68.0 \%)$ \\
\hline $\begin{array}{l}\text { Muscle pain and } \\
\text { weakness }\end{array}$ & $28 / 163(17.2 \%)$ & $4 / 25(16.0 \%)$ \\
\hline Financial reasons & $6 / 163(3.7 \%)$ & $0 / 25(0 \%)$ \\
\hline $\begin{array}{l}\text { Modified dose } \\
\text { and/or } \\
\text { frequency }\end{array}$ & 214/1244 (17.8\%) & $59 / 307(19.2 \%)$ \\
\hline $\begin{array}{l}\text { Insufficient lipid- } \\
\text { lowering effect }\end{array}$ & $141 / 214(65.9 \%)$ & $45 / 59(76.3 \%)$ \\
\hline $\begin{array}{l}\text { Muscle pain and } \\
\text { weakness }\end{array}$ & $19 / 214(8.9 \%)$ & $6 / 59(10.2 \%)$ \\
\hline Financial reasons & $6 / 214(2.8 \%)$ & $3 / 59(5.1 \%)$ \\
\hline $\begin{array}{l}\text { Increased liver } \\
\text { enzymes }\end{array}$ & $6 / 214(2.8 \%)$ & $0 / 59(0 \%)$ \\
\hline Discontinued & $65 / 1244(5.4 \%)$ & $22 / 307(7.2 \%)$ \\
\hline $\begin{array}{l}\text { Muscle pain and } \\
\text { weakness }\end{array}$ & $19 / 65(29.2 \%)$ & $13 / 22(59.1 \%)$ \\
\hline $\begin{array}{l}\text { Insufficient lipid- } \\
\text { lowering effect }\end{array}$ & $10 / 65(15.4 \%)$ & $3 / 22(13.6 \%)$ \\
\hline
\end{tabular}

${ }^{a}$ Reasons are expressed as \% of patients in each status

\section{Changes in Lipid-Modifying Therapies}

As summarised in Table 5, a total of 163 patients (13.6\%) switched to other LMT, 214 (17.8\%) had modifications in dose and/or frequency and $65(5.4 \%)$ discontinued LMT. In the FH subgroup, $8.1 \%$ switched to other LMT, $19.2 \%$ had modifications in dose and/or frequency and $7.2 \%$ discontinued their LMT. The most common reasons for these changes were insufficient 
lipid-lowering effect and muscle pain and weakness.

\section{DISCUSSION}

This observational study explored how elevated LDL-C in HR and VHR patients with dyslipidaemia is currently managed in central/eastern Europe and Israel. Our study population consisted mainly of VHR/secondary prevention patients attending specialist clinics; approximately $40 \%$ were diabetic and one-quarter had FH. Less than one-quarter $(23.8 \%)$ of the HR patients and less than half $(42.0 \%)$ of the VHR patients achieved their risk-based LDL-C targets of $<2.5$ and $<1.8 \mathrm{mmol} / \mathrm{L}$, respectively. The revised (2016) ESC/EAS target for HR patients $(2.6 \mathrm{mmol} / \mathrm{L})$ was met by $28.5 \%$ of our HR patients.

Our findings are consistent with numerous previous reports, from various geographical regions, of low rates of LDL-C target achievement in patients with hyperlipidaemia [14-17]. For instance, the EUROASPIRE IV survey of 6648 coronary heart disease (CHD) patients at 79 centres (largely academic hospitals) in 24 European countries found that at follow-up $\geq 6$ months after hospital discharge, LDL-C values were $<2.5 \mathrm{mmol} / \mathrm{L}$ in $57.8 \%$ of patients and $<1.8 \mathrm{mmol} / \mathrm{L}$ in $19.3 \%$ of patients [14]. Similarly, the Dyslipidaemia International study (DYSIS), a survey of almost 58,000 statintreated patients across Europe, the Middle East, Canada, Africa and Asia, found that only $21.7 \%$ of the VHR patients and $38.0 \%$ of the HR patients attained their LDL-C goals [15].

Fewer than $15 \%$ of our FH patients reached their LDL-C goals. Approximately half had probable or definite FH by DLCN criteria $[1,12]$ (score $\geq 6$ ), which are based not only on LDL-C but also on family and clinical history and physical examination. FH diagnosis based on clinical criteria may lead to both false positive and false negative diagnoses when matched against genetic analysis, often reflecting polygenic, rather than monogenic, severe hypercholesterolaemia. For untreated patients with definite or probable $\mathrm{FH}$, the risk of $\mathrm{CHD}$ is estimated to be $\geq 10$-fold higher than the general population but can be substantially reduced with timely cascade screening and appropriate treatment. However, in practice it is an underdiagnosed and undertreated disease [18-20]. For instance, a Danish population survey in $>69,000$ individuals found that only $48 \%$ of those with FH were receiving LMT. The adjusted odds ratio for coronary artery disease in those with definite/probable FH versus nonFH subjects was $13.2(10.0-17.4)$ without LMT and 10.3 (7.8-13.8) with LMT [20].

Multiple issues may potentially contribute to inadequate LDL-C reduction in patients with hyperlipidaemia. Underdosing and statin discontinuation/poor adherence to therapy are acknowledged as key contributors and have been linked to poorer clinical outcomes $[8,13,21]$. Statin nonadherence is a challenge for clinicians and generally worsens over treatment duration, with many reports of adherence rates $<50 \%$, particularly in primary prevention settings [8, 21-23]. As discontinuation of statins and poor patient adherence to treatment can be related to SAMS, detailed expert guidelines for diagnosing and managing this condition have recently been published by an EAS Consensus Panel [13].

Financial/reimbursement issues may be an additional factor. Some countries have only partial reimbursement for drug treatment (e.g. Bulgaria with 25\% reimbursement and $75 \%$ copayment) which, together with low disposable income, can result in patients being restricted to cheaper treatments/lower doses. Ezetimibe is not reimbursed in some countries and this may have been a factor in the low usage of statin/ ezetimibe combinations. Healthcare systems in the eastern Europe region are based on the reference pricing principle and therefore much more restrictive in permitting newer treatments, waiting for the largest markets to reimburse the new products and deliver data showing real-world results from the change in clinical practice.

Only half of our patients (53\%) overall were taking high-intensity statin therapy and only $13 \%$ were receiving statin + ezetimibe combinations. Approximately $60-70 \%$ of HR and $60 \%$ of VHR patients who did not receive high-intensity statins did not achieve their target 
during observation. Overall, $10 \%$ of patients were listed as having statin intolerance symptoms, most commonly SAMS (8\% of patients). Despite most patients not reaching their LDL-C targets, only $14 \%$ switched to another LMT during the observation period, while $18 \%$ had modifications in dose and/or frequency, generally because of insufficient lipid-lowering or muscle pain/weakness. Only $5 \%$ of patients discontinued LMT. Financial reasons were cited as the reason for changing treatment in six patients (3.7\%).

If LDL-C is extremely elevated, as is often the case in FH patients, even maximal-dosage statins plus ezetimibe may not be able to lower LDL-C to within the target range. Alternative treatments for hyperlipidaemia are urgently needed and are currently being developed.

Only $2 \%$ of our patients (mostly those in the SI group; $n=22$ ) were receiving PCSK9is, which were only just becoming available in many of the participating countries at the time the study was being conducted. This class of drugs target the serine protease PCSK9, which plays a key role in LDL receptor (LDLR) activity [24]: inhibiting PCSK9 results in improved LDLR recycling and increased LDLR availability on hepatocyte cell surfaces. When added to existing maximally tolerated statin therapy, the monoclonal antibodies alirocumab and evolocumab can effect further LDL-C reductions of up to $60 \%$, leading to target levels being met in up to $80 \%$ of patients [25-29]. Recently published outcome data from the FOURIER and ODYSSEY studies indicate that evolocumab (Repatha) [27] and alirocumab (Praluent) [30] significantly reduce the risk of CV events. Their safety profile differs substantially from that of statins, with no evidence of creatine kinase elevation, myalgia/muscle symptoms and few patients discontinuing treatment because of adverse events [26, 27, 29]. Further long-term safety data are awaited with interest. Recently updated guidelines from an ESC/EAS Task Force indicate that PCSK9i may be considered for VHR patients with atherosclerotic CVD (ASCVD), including those with progressive ASCVD or diabetes with target organ damage or a major $\mathrm{CV}$ risk factor or severe $\mathrm{FH}$ without
ASCVD but severely elevated LDL-C despite maximal statin/ezetimibe therapy [31].

Almost half of our patients (42.1\%) experienced one or more CV events during observation. This very high rate of events includes nonmajor events and reflects the nature of our study population, as well as the very high rate of $\mathrm{CV}$ events and mortality seen in many developing European countries [32].

Our observational study has some limitations. The study population was drawn mainly from cardiology, lipidology and diabetic clinics, and the mix of centres and patients differed between the participating countries, which limits the generalisability of our results. Patients had a variable duration of LMT, with approximately $20 \%$ being newly diagnosed. They were treated and monitored according to local clinical practice, with no prestipulated LDL-C measurement intervals. Lipid measurements were conducted at local laboratories; thus, there will be some differences due to assay biases between laboratories. As previously mentioned, the diagnosis of FH was uncertain for some patients. Nevertheless, our data provide a useful snapshot of hyperlipidaemia management patterns throughout the region. To our knowledge this is the first systematic study conducted in eastern Europe and Israel, other than EUROASPIRE [14].

\section{CONCLUSIONS}

Our findings provide real-world evidence from current clinical practice in eastern/central Europe and Israel. They confirm reports from other geographical areas that, despite widespread use of statins, many patients being treated for hyperlipidaemia, particularly those with $\mathrm{FH}$, have persistent above-target LDL-C levels, thus remaining at risk of $\mathrm{CV}$ events. Further efforts are needed to address the unmet needs in these patients.

\section{ACKNOWLEDGEMENTS}

The authors wish to thank the staff and patients of the participating clinics. 
Funding. Amgen (Europe) $\mathrm{GmbH}$ designed the study in cooperation with the authors and provided financial support for the study, statistical analysis and manuscript, including article processing charges and the open access fee.

Medical Writing and Other Assistance. Julia Balfour of Northstar Medical Writing and Editing, Dundee, UK, drafted and revised the manuscript together with the authors, with financial support from Amgen. Joshua David and Mohamed Riaz Anwar of Quartesian, Bangalore, provided the statistical analysis.

Authorship. All named authors meet the International Committee of Medical Journal Editors (ICMJE) criteria for authorship for this article, take responsibility for the integrity of the work as a whole, and have given their approval for this version to be published.

Authorship Contributions. Ivo Petrov, Beata Wożakowska-Kapłon, Hrvoje Pintarić, Ian Bridges and Reneta Petkova contributed to conception and/or or design of the study. All authors contributed to acquisition, analysis, and/or interpretation of data for the work, critically revised the manuscript, gave final approval and agree to be accountable for all aspects of work ensuring integrity and accuracy.

Disclosures. Ian Bridges is an employee and stockholder of Amgen. Reneta Petkova is an employee and stockholder of Amgen. Ivo Petrov has received research grants from Quintiles, consulting fees from Amgen, Actavis, Novartis, Medtronic, MSD, Boehringer Ingelheim, Pfizer and speaker honoraria from Amgen, Minvasys and Contego Medical. Michaela Snejdrlova discloses honoraria as a speaker for Amgen, MSD, Servier and Mylan and Research support from the Czech Ministry of Health. Lubomira Fabryova has received consulting fees from Abbott, Amgen, Boehringer Ingelheim, Eli Lilly, MSD, Novo Nordisk, Sanofi Aventis, Servier, Valeant and speaker honoraria from Amgen, MSD, Sanofi Aventis and Valeant. Barak Zafrir has received consulting fees from Medison Israel and Sanofi Israel. Andreea Dumitrescu, Beata
Wożakowska-Kapłon and Hrvoje Pintarić have nothing to disclose.

Compliance with Ethics Guidelines. There were no study-related medical procedures undertaken. The study was conducted in accordance with the Helsinki Declaration of 1964, as revised in 2013, and with countryspecific legal and regulatory requirements. It was approved by ethics committees and for registration or classification by regulatory bodies, as applicable in each country:

1. Bulgaria: Republic of Bulgaria Ministry of Health Ethics Committee for Multicentre Trial, Sofia; 2. Croatia: Agency for Medicinal Products and Medical Products Central Ethics Trust, Zagreb; 3. Czech Republic: Ethics Committee of: Faculty Hospital Hradec Králové, Králové; Institute for Clinical and Experimental Medicine (IKEM) and Thomayer Hospital, Prague; Faculty Hospital Brno, Brno; Faculty Hospital Plzeň, Plzeň; General Teaching Hospital in Prague, Prague; St. Anne's Faculty Hospital in Brno, Brno; Ethics Committee for Multicentre Clinical Assessment of the Motol University Hospital, Prague; Pardubice Regional Hospital Svitavska Hospital 4. Israel: Helsinki Committees of: Edith Wolfson Medical Centre, Tel Aviv; Chaim Sheba Medical Center, Tel Hashomer; Hadassah Medical Center, Jerusalem; Rabin Medical Center, Petah Tikva; Lady Davis Carmel Medical Center, Haifa; Soroka Medical Center, Beersheba; Bnai Zion Medical Center, Haifa; Meir Medical Center, Kfar Saba; Ziv Medical Center, Safed. 5. Poland: Bioethical Commission in Kielce at the Świętokrzyska Medical Chamber. 6. Romania: Romania Medical Sciences Academy, National Bioethics Committee for Medicines and Medical Devices, Bucharest. 7. Slovakia: Multicentre Ethics Committee of Kosice Self-Governing Region, Kosice.

The study followed generally accepted research practices described in Good Epidemiological Practice guidelines issued by the International Epidemiological Association. All data were handled in strictest confidence in conformity with national and European data protection regulations (such as Directive 95/46/EC). All patients provided written informed consent, where required by local regulations. 
Data Availability. Data sharing requests relating to data in this manuscript will be considered after the publication date.

There is no end date for eligibility to submit a data sharing request for these data. Qualified researchers may submit a request containing the research objectives, endpoints/outcomes of interest, statistical analysis plan, data requirements, publication plan, and qualifications of the researcher(s). In general, Amgen does not grant external requests for individual patient data for the purpose of re-evaluating safety and efficacy issues already addressed in the product labelling. A committee of internal advisors reviews requests.

Requests that pose a potential conflict of interest or an actual or potential competitive risk may be declined at Amgen's sole discretion and without further arbitration. Upon approval, information necessary to address the research question will be provided under the terms of a data sharing agreement. This may include anonymized individual patient data and/or available supporting documents, containing fragments of analysis code where provided in analysis specifications. Further details are available at the following: http://www.amgen.com/ datasharing.

Open Access. This article is distributed under the terms of the Creative Commons Attribution-NonCommercial 4.0 International License (http://creativecommons.org/licenses/ by-nc/4.0/), which permits any noncommercial use, distribution, and reproduction in any medium, provided you give appropriate credit to the original author(s) and the source, provide a link to the Creative Commons license, and indicate if changes were made.

\section{REFERENCES}

1. World Health Organization: Cardiovascular diseases. Fact sheet. Updated May 2017. http://www. who.int/mediacentre/factsheets/fs317/en/; 2017. Accessed 25 Jan 2019.

2. Tolonen H, Keil U, Ferrario M, Evans A. Prevalence, awareness and treatment of hypercholesterolaemia in 32 populations: results from the WHO MONICA Project. Int J Epidemiol. 2005;34(1):181-92.

3. Kannel WB. The Framingham Study: an epidemiological investigation of cardiovascular disease, Section 30. Some characteristics related to the incidence of cardiovascular disease and death: the Framingham Study. 18-year follow-up. Washington, D.C.: Dept. of Health, Education, and Welfare, Publication No. (NIH) 74-599; 1974.

4. Kannel WB. Range of serum cholesterol values in the population developing coronary artery disease. Am J Cardiol. 1995;76(9 Suppl. 1):69C-77C.

5. Kannel WB, Castelli WP, Gordon T. Cholesterol in the prediction of atherosclerotic disease: new perspectives based on the Framingham study. Ann Intern Med. 1979;90(1):85-91.

6. Cholesterol Treatment Trialists Collaboration. Efficacy and safety of more intensive lowering of LDL cholesterol: a meta-analysis of data from 170,000 participants in 26 randomised trials. Lancet. 2010;376(9753):1670-81.

7. Reiner Z, Catapano AL, De Backer G, et al. ESC/EAS Guidelines for the management of dyslipidaemias: the Task Force for the management of dyslipidaemias of the European Society of Cardiology (ESC) and the European Atherosclerosis Society (EAS). Eur Heart J. 2011;32(14):1769-818.

8. Catapano AL, Graham I, De Backer G, et al. 2016 ESC/EAS guidelines for the management of dyslipidaemias: the Task Force for the Management of Dyslipidaemias of the European Society of Cardiology (ESC) and European Atherosclerosis Society (EAS) developed with the special contribution of the European Association for Cardiovascular Prevention and Rehabilitation (EACPR). Atherosclerosis. 2016;253:281-344.

9. Pajak A, Kozela M. Cardiovascular disease in central and east Europe. Public Health Rev. 2012;33: 416-35.

10. Eurostat. Eurostat online data: deaths from cardiovascular diseases. Eurostat. 2018. http://ec.europa. eu/eurostat/statistics-explained/index.php?title= Cardiovascular_diseases_statistics. Accessed 1 Jan 2019.

11. Banach M, Rizzo M, Toth PP, et al. Statin intolerance-an attempt at a unified definition. Position paper from an International Lipid Expert Panel. Expert Opin Drug Saf. 2015;14(6):935-55.

12. Hovingh GK, Davidson MH, Kastelein JJ, O'Connor AM. Diagnosis and treatment of familial hypercholesterolaemia. Eur Heart J. 2013;34(13):962-71. 
13. Stroes ES, Thompson PD, Corsini A, et al. Statinassociated muscle symptoms: impact on statin therapy-European Atherosclerosis Society Consensus Panel Statement on Assessment, Aetiology and Management. Eur Heart J. 2015;36(17): 1012-22.

14. Reiner Ž, De Backer G, Fras Z, et al. Lipid lowering drug therapy in patients with coronary heart disease from 24 European countries-findings from the EUROASPIRE IV survey. Atherosclerosis. 2016;246:243-50.

15. Gitt AK, Lautsch D, Ferrieres J, et al. Low-density lipoprotein cholesterol in a global cohort of 57,885 statin-treated patients. Atherosclerosis. 2016;255: 200-9.

16. Zafrir B, Jubran A, Lavie G, Halon DA, Flugelman MY, Shapira C. Clinical determinants and treatment gaps in familial hypercholesterolemia: data from a multi-ethnic regional health service. Eur J Prev Cardiol. 2017;24(8):867-75.

17. Perez de Isla L, Alonso R, Watts GF, et al. Attainment of LDL-cholesterol treatment goals in patients with familial hypercholesterolemia: 5-year SAFEHEART Registry follow-up. J Am Coll Cardiol. 2016;67(11):1278-85.

18. Marks D, Thorogood M, Neil HA, Humphries SE. A review on the diagnosis, natural history, and treatment of familial hypercholesterolaemia. Atherosclerosis. 2003;168(1):1-14.

19. Nordestgaard BG, Chapman MJ, Humphries SE, et al. Familial hypercholesterolaemia is underdiagnosed and undertreated in the general population: guidance for clinicians to prevent coronary heart disease Consensus Statement of the European Atherosclerosis Society. Eur Heart J. 2013;34(45):3478-90.

20. Benn M, Watts GF, Tybjaerg-Hansen A, Nordestgaard BG. Familial hypercholesterolemia in the Danish general population: prevalence, coronary artery disease, and cholesterol-lowering medication. J Clin Endocrinol Metab. 2012;97(11): 3956-64.

21. Banach M, Stulc T, Dent R, Toth PP. Statin nonadherence and residual cardiovascular risk: there is need for substantial improvement. Int J Cardiol. 2016;225:184-96.

22. Perreault S, Blais L, Dragomir A, et al. Persistence and determinants of statin therapy among middleaged patients free of cardiovascular disease. Eur J Clin Pharmacol. 2005;61(9):667-74.
23. Blackburn DF, Dobson RT, Blackburn JL, Wilson TW, Stang MR, Semchuk WM. Adherence to statins, beta-blockers and angiotensin-converting enzyme inhibitors following a first cardiovascular event: a retrospective cohort study. Can J Cardiol. $2005 ; 21(6): 485-8$.

24. Seidah NG, Awan Z, Chrétien M, Mbikay M. PCSK9: a key modulator of cardiovascular health. Circ Res. 2014;114(6):1022-36.

25. Lipinski MJ, Benedetto U, Escarcega RO, et al. The impact of proprotein convertase subtilisin-kexin type 9 serine protease inhibitors on lipid levels and outcomes in patients with primary hypercholesterolaemia: a network meta-analysis. Eur Heart J. 2016;37(6):536-45.

26. Navarese EP, Kolodziejczak M, Schulze V, et al. Effects of proprotein convertase subtilisin/kexin type 9 antibodies in adults with hypercholesterolemia: a systematic review and meta-analysis. Ann Intern Med. 2015;163(1):40-51.

27. Sabatine MS, Giugliano RP, Keech AC, et al. Evolocumab and clinical outcomes in patients with cardiovascular disease. N Engl J Med. 2017;376(18): 1713-22.

28. Sabatine MS, Giugliano RP, Pedersen TR. Evolocumab in patients with cardiovascular disease. $\mathrm{N}$ Engl J Med. 2017;377(8):787-8.

29. Sabatine MS, Giugliano RP, Wiviott SD, et al. Efficacy and safety of evolocumab in reducing lipids and cardiovascular events. $\mathrm{N}$ Engl J Med. 2015;372(16):1500-9.

30. Robinson JG, Farnier M, Krempf M, et al. Efficacy and safety of alirocumab in reducing lipids and cardiovascular events. $\mathrm{N}$ Engl J Med. 2015;372(16):1489-99.

31. Landmesser U, Chapman MJ, Stock JK, et al. 2017 update of ESC/EAS Task Force on practical clinical guidance for proprotein convertase subtilisin/kexin type 9 inhibition in patients with atherosclerotic cardiovascular disease or in familial hypercholesterolaemia. Eur Heart J. 2018;39:1131-43.

32. OECD/EU. Health at a glance: Europe 2016 state of health in the EU cycle.OECD Publishing. 2016. https:/ec.europa.eu/health/sites/health/files/state/ docs/health_glance_2016_rep_en.pdf. Accessed 25 Jan 2019. 